\title{
Cellulose-Sulfuric Acid as an Efficient Biosupported Catalyst in One-Pot Synthesis of Novel Heteroaryl Substituted 1,4-Dihydropyridines
}

\author{
Manouchehr Mamaghani, Khalil Tabatabaeian, \\ Mohaddesseh Mohammadi, and Alireza Khorshidi \\ Department of Chemistry, Faculty of Sciences, University of Guilan, P.O. Box 41335-1914, Rasht, Iran \\ Correspondence should be addressed to Manouchehr Mamaghani; mchem41@gmail.com
}

Received 23 May 2013; Accepted 3 September 2013

Academic Editor: Mohamed Afzal Pasha

Copyright (c) 2013 Manouchehr Mamaghani et al. This is an open access article distributed under the Creative Commons Attribution License, which permits unrestricted use, distribution, and reproduction in any medium, provided the original work is properly cited.

\begin{abstract}
An efficient method for the synthesis of new heteroaryl substituted dihydropyridine derivatives via a one-pot four-component coupling reaction of heteroaldehyde, 1,3-diketone, ethylacetoacetate, and amonium acetate in the presence of cellulose-sulfuric acid as a biosupported solid acid catalyst was developed. The reaction gave the new derivatives of fused 1,4-dihydropyridines in lower reaction times and excellent yields (85-95\%).
\end{abstract}

\section{Introduction}

1,4-Dihydropyridines exhibit interesting pharmacological and biological properties. For example, they have been used as calcium channel modulators for the treatment of cardiovascular disorders [1, 2]. They also present vasodilating [3], antifilarial [4], and antitubercular activities [5] and may serve as NADH mimics [6,7]. Many methods have been reported for the synthesis of 1,4-dihydropyridine derivatives in view of the biological importance associated with these compounds. The best known procedure for preparation of symmetrical 1,4-dihydropyridines is the classical Hantzch synthesis: a multicomponent condensation involving two molecules of $\beta$-ketoester, one molecule of aldehyde, and one molecule of ammonia $[8,9]$. Other reported methods comprise the use of microwave [10-12], ionic liquids [13, 14], high temperature in refluxing solvent [15-24], TMSCl-NaI [25], and metal triflates $[26,27]$, which have their own drawbacks such as high temperature, expensive metal precursors, and prolonged reaction times. Thus, development of a simple, efficient, and versatile method for preparation of new derivatives of 1,4dihydropyridine is a great challenge.

\section{Experimental}

2.1. General. Melting points were measured on an electrothermal 9100 apparatus and are uncorrected. ${ }^{1} \mathrm{H}$ NMR spectra were obtained on a Bruker DRX-500 Avance spectrometer, and ${ }^{13} \mathrm{C}$ NMR spectra were obtained on a Bruker DRX-125 Avance spectrometer. Chemical shifts of ${ }^{1} \mathrm{H}$ and ${ }^{13} \mathrm{C}$ NMR spectra were expressed in ppm downfield from tetramethylsilane. FT-IR spectra were recorded on a Shimadzu FT-IR-8400S spectrometer. Chemicals were purchased from Merck and Fluka and used without further purification.

2.2. Typical Procedure: Preparation of 3-Aryl-4-fromyl-1-phenylpyrazole. In a round bottomed flask, cyanuric chloride $(1.83 \mathrm{~g}, 10 \mathrm{mmol})$ was added to dimethyl formamide $(2 \mathrm{~mL})$ which resulted in a white solid. To this product a solution of phenylhydrazone acetophenone (1 g, $5 \mathrm{mmol})$ in dimethyl formamide $(15 \mathrm{~mL})$ was added and stirred for $16 \mathrm{~h}$ at room temperature. To the resulted mixture, $15 \% \mathrm{Na}_{2} \mathrm{CO}_{3}(20 \mathrm{~mL})$ was added and the organic layer was extracted by diethylether $(2 \times 15 \mathrm{~mL})$. The ethereal solution was dried by $\mathrm{MgSO}_{4}$ and filtered, and the filtrate evaporated under vacuum to 
TABLE 1: Synthesis of heteroaryl substituted 1,4-dihydropyridines in the presence of cellulose-sulfuric acid catalyst.

\begin{tabular}{|c|c|c|c|c|c|}
\hline Entry $^{a}$ & $\mathrm{Ar}$ & 2 & Time (min) & Product & Yield $(\%)^{\mathrm{b}}$ \\
\hline a & $\mathrm{Ph}$ & Dimedone & $42(120)^{c}$ & $5 a$ & $90(75)^{c}$ \\
\hline b & $p-\mathrm{NO}_{2} \mathrm{C}_{6} \mathrm{H}_{4}$ & Dimedone & 45 & $5 \mathbf{b}$ & 95 \\
\hline c & $p-\mathrm{ClC}_{6} \mathrm{H}_{4}$ & Dimedone & 40 & $5 c$ & 94 \\
\hline d & $\mathrm{Ph}$ & 1,3-Cyclohexanedione & $43(125)^{\mathrm{c}}$ & $5 d$ & $88(72)^{\mathrm{c}}$ \\
\hline e & $p-\mathrm{NO}_{2} \mathrm{C}_{6} \mathrm{H}_{4}$ & 1,3-Cyclohexanedione & 45 & $5 e$ & 87 \\
\hline f & $p-\mathrm{ClC}_{6} \mathrm{H}_{4}$ & 1,3-Cyclohexanedione & 42 & $5 f$ & 86 \\
\hline g & $\mathrm{Ph}$ & 1,3-Indanedione & $39(120)^{\mathrm{c}}$ & $5 g$ & $90(75)^{\mathrm{c}}$ \\
\hline h & $p-\mathrm{NO}_{2} \mathrm{C}_{6} \mathrm{H}_{4}$ & 1,3-Indanedione & 41 & $5 \mathrm{~h}$ & 86 \\
\hline $\mathbf{i}$ & $p-\mathrm{ClC}_{6} \mathrm{H}_{4}$ & 1,3-Indanedione & 37 & $5 \mathbf{i}$ & 85 \\
\hline
\end{tabular}

${ }^{\mathrm{a}}$ All products were characterized by ${ }^{1} \mathrm{H} \mathrm{NMR},{ }^{13} \mathrm{C} \mathrm{NMR}$, and IR data.

${ }^{\mathrm{b}}$ Isolated yields.

${ }^{c}$ Measured in the absence of the catalyst.

produce 1,3-diphenyl-4-formylpyrazole (1.12 g) in 90\% yield. The product was identified by spectroscopic analysis and comparison of its melting point $\left(\mathrm{mp}=146-148^{\circ} \mathrm{C}\right)$ with the reported one $\left(\mathrm{mp}=145-147^{\circ} \mathrm{C}\right)[28]$.

Light yellow solid, IR $(\mathrm{KBr})\left(v_{\max } / \mathrm{cm}^{-1}\right): 2850,2750(\mathrm{H}-$ $\mathrm{C}=\mathrm{O}), 1670$ (C=O), 1590, 1520, 750, 690. ${ }^{1} \mathrm{H}$ NMR $(500 \mathrm{MHz}$, $\left.\mathrm{CDCl}_{3}\right): \delta 10.11(1 \mathrm{H}, \mathrm{s}), 8.59(1 \mathrm{H}, \mathrm{s}), 7.89-7.84(4 \mathrm{H}, \mathrm{m})$, $7.58-7.52(5 \mathrm{H}, \mathrm{m}), 7.44(1 \mathrm{H}, \mathrm{t}, J=7.44 \mathrm{~Hz}) \mathrm{ppm} ;{ }^{13} \mathrm{C} \mathrm{NMR}$ $\left(125 \mathrm{MHz} \mathrm{CDCl}_{3}\right) \delta 185.6,155.2,139.5,131.8,131.4,130.1,129.7$, $129.4,129.2,128.4,123.0,120.2 \mathrm{ppm}$.

2.2.1. 3-(4-Nitrophenyl)-1-phenyl-1H-pyrazole-4-carbaldehyde. Yellow solid; FT-IR (neat) $\left(v_{\max } / \mathrm{cm}^{-1}\right): 1680(\mathrm{HC}=\mathrm{O})$, 1520, $1340\left(\mathrm{NO}_{2}\right) .{ }^{1} \mathrm{H} \mathrm{NMR}\left(500 \mathrm{MHz}, \mathrm{CDCl}_{3}\right) \delta 7.49(1 \mathrm{H}, \mathrm{t}$, $J=7.40 \mathrm{~Hz}), 7.59(2 \mathrm{H}, \mathrm{t}, J=7.90 \mathrm{~Hz}), 7.85(2 \mathrm{H}, \mathrm{d}, J=7.74 \mathrm{~Hz})$, $8.21(2 \mathrm{H}, \mathrm{d}, J=8.70 \mathrm{~Hz}), 8.38(2 \mathrm{H}, \mathrm{d}, J=8.70 \mathrm{~Hz}), 8.62$ $(1 \mathrm{H}, \mathrm{s}), 10.13(1 \mathrm{H}, \mathrm{s}) ;{ }^{13} \mathrm{C} \mathrm{NMR}\left(125 \mathrm{MHz}, \mathrm{CDCl}_{3}\right) \delta 184.0$ $(\mathrm{HC}=\mathrm{O}), 151.7,148.6,139.1,138.1,134.0,130.3,130.1,128.9$, $124.2,123.4,120.2$.

2.3. General Procedure for the Synthesis of 1,4-Dihydropyridines $(\mathbf{5} \boldsymbol{a}-\mathbf{i})$. A mixture of 3-aryl-4-formylpyrazole (1) (1.0 mmol), 1,3-diketone (2) (1.0 mmol, dimedone or 1,3cyclohexanedione or indanedione), ethylacetoacetate (3) $(1.0 \mathrm{mmol})$, ammonium acetate $(1.0 \mathrm{mmol})$, and cellulosesulfuric acid $(0.05 \mathrm{~g})$ in ethanol $(10 \mathrm{~mL})$ was refluxed in an oil bath for the appropriate time (Table 1). The progress of the reaction was controlled by TLC, and after completion of the reaction the solvent was evaporated under reduced pressure to provide a residue which was purified by recrystallization from ethanol to furnish the desired 1,4-dihydropyridines (5ai).

2.3.1. Ethyl 4-(1,3-Diphenyl-1H-pyrazol-4-yl)-2,7,7-trimethyl5-oxo-1,4,5,6,7,8-hexahydro quinoline-3-carboxylate (5a). Yellow powder, $\mathrm{mp}=106-108^{\circ} \mathrm{C}$; FT-IR (neat) $\left(v_{\max } / \mathrm{cm}^{-1}\right)$ 3300, $3200(\mathrm{NH}), 1695$ (C=O), 1635 (C=C, olefinic); ${ }^{1} \mathrm{H}$ NMR $\left(500 \mathrm{MHz} \mathrm{CDCl}_{3}\right) \delta 7.95(2 \mathrm{H}, \mathrm{d}, J=7.32 \mathrm{~Hz}), 7.81(1 \mathrm{H}, \mathrm{s}) 7.69$ $(2 \mathrm{H}, \mathrm{d}, J=7.90 \mathrm{~Hz}), 7.41-7.47(4 \mathrm{H}, \mathrm{m}), 7.37(1 \mathrm{H}, \mathrm{t}, J=7.38 \mathrm{~Hz})$, $7.26(1 \mathrm{H}, \mathrm{t}, J=7.38 \mathrm{~Hz}), 6.03(1 \mathrm{H}, \mathrm{s}), 5.32(1 \mathrm{H}, \mathrm{s}), 3.64,3.97$ $(2 \mathrm{H}, \mathrm{m}), 2.21,2.25(2 \mathrm{H}, \mathrm{d}, J=16.36 \mathrm{~Hz}), 2.19(3 \mathrm{H}, \mathrm{s}), 2.13$,
$2.23(2 \mathrm{H}, \mathrm{d}, J=16.50 \mathrm{~Hz}), 1.08(3 \mathrm{H}, \mathrm{s}), 1.04(3 \mathrm{H}, \mathrm{s}), 0.92(3 \mathrm{H}$, $\mathrm{t}, J=7.09 \mathrm{~Hz}) ;{ }^{13} \mathrm{C}$ NMR $\left(125 \mathrm{MHz}, \mathrm{CDCl}_{3} / \mathrm{DMSO}_{-} \mathrm{d}_{6}\right) \delta$ 196.0 ( $\mathrm{C}=\mathrm{O}$, ketone), 167.8 ( $\mathrm{C}=\mathrm{O}$, ester), 152.2, 148.5, 142.8, $140.5,135.2,129.7,129.6,128.6,128.3,127.9,127.5,126.4,119.3$, 112.3, 107.1, 60.0, 51.1, 41.5, 33.1, 30.1, 28.3, 27.4, 19.6, 14.4. Anal. Calcd for $\mathrm{C}_{30} \mathrm{H}_{31} \mathrm{~N}_{3} \mathrm{O}_{3}$ : C, 74.82; $\mathrm{H}, 6.49 ; \mathrm{N}, 8.73$. Found: C, $74.73 ; \mathrm{H}, 6.26$; N, 8.56.

2.3.2. Ethyl 2,7,7-Trimethyl-4-(3-(4-nitrophenyl)-1-phenyl1H-pyrazol-4-yl)-5-oxo-1,4,5,6,7,8-hexahydroquinoline-3-carboxylate $(\mathbf{5 b})$. Yellow solid $\mathrm{mp}=256-258^{\circ} \mathrm{C}$; FT-IR (neat) $\left(v_{\max } / \mathrm{cm}^{-1}\right)$ 3250, $3200(\mathrm{NH}), 1695(\mathrm{C}=\mathrm{O}), 1650(\mathrm{C}=\mathrm{C}$, olefinic), 1510, $1330\left(\mathrm{NO}_{2}\right) ;{ }^{1} \mathrm{H}$ NMR $\left(500 \mathrm{MHz}, \mathrm{CDCl}_{3}\right) \delta$ $8.44(2 \mathrm{H}, \mathrm{d}, J=8.88 \mathrm{~Hz}), 8.38(2 \mathrm{H}, \mathrm{d}, J=8.88 \mathrm{~Hz}), 7.80(1 \mathrm{H}$, s), $7.70(2 \mathrm{H}, \mathrm{d}, J=7.62 \mathrm{~Hz}), 7.47(2 \mathrm{H}, \mathrm{t}, J=7.95 \mathrm{~Hz}), 7.32$ $(1 \mathrm{H}, \mathrm{t}, J=7.40 \mathrm{~Hz}), 5.86(1 \mathrm{H}, \mathrm{s}), 5.33(1 \mathrm{H}, \mathrm{s}), 3.95,3.63(2 \mathrm{H}$, $\mathrm{m}), 2.41,2.25(2 \mathrm{H}, \mathrm{d}, J=16.46 \mathrm{~Hz}), 2.33(3 \mathrm{H}, \mathrm{s}), 2.25,2.32$ $(2 \mathrm{H}, \mathrm{d}, J=16.30 \mathrm{~Hz}), 1.15(3 \mathrm{H}, \mathrm{s}), 1.07(3 \mathrm{H}, \mathrm{s}), 0.86(3 \mathrm{H}, \mathrm{t}, J$ $=7.12 \mathrm{~Hz}) ;{ }^{13} \mathrm{C}$ NMR $\left(125 \mathrm{MHz}, \mathrm{CDCl}_{3} / \mathrm{DMSO}_{6}\right) \delta 196.1$ $(\mathrm{C}=\mathrm{O}), 167.4(\mathrm{C}=\mathrm{O}$, ester $), 149.2,148.0,147.5,142.7,142.0$, $140.3,130.0,129.8,128.2,127.0,123.8,119.5,112.9,107.7,60.2$, 51.0, 41.8, 33.3, 29.5, 28.0, 27.4, 20.0, 14.4. Anal. Calcd for $\mathrm{C}_{30} \mathrm{H}_{30} \mathrm{~N}_{4} \mathrm{O}_{5}$ : C, 68.42; H, 5.74; N, 10.64. Found: C, 68.25; H, $5.64 ; \mathrm{N}, 10.75$.

2.3.3. Ethyl 4-(3-(4-Chlorophenyl)-1-phenyl-1H-pyrazol-4-yl)2,7,7-trimethyl-5-oxo-1,4,5,6,7,8-hexahydroquinoline-3-carboxylate $(5 \mathrm{c})$. White solid, $\mathrm{mp}=253-255^{\circ} \mathrm{C}$; FT-IR (neat) $\left(v_{\max } / \mathrm{cm}^{-1}\right): 3300,3200(\mathrm{NH}), 1690(\mathrm{C}=\mathrm{O}), 1640(\mathrm{C}=\mathrm{C}$, olefinic), 1080 (C-Cl); ${ }^{1} \mathrm{H} \mathrm{NMR}\left(500 \mathrm{MHz}, \mathrm{CDCl}_{3}\right) \delta 7.98$ $(2 \mathrm{H}, \mathrm{d}, J=8.20 \mathrm{~Hz}), 7.97(1 \mathrm{H}, \mathrm{s}), 7.66(1 \mathrm{H}, \mathrm{s}), 7.54(2 \mathrm{H}, \mathrm{d}, J=$ $7.55 \mathrm{~Hz}), 7.32(2 \mathrm{H}, \mathrm{d}, J=8.20 \mathrm{~Hz}), 7.30(2 \mathrm{H}, \mathrm{m} 2 \mathrm{H}), 7.14(1 \mathrm{H}$, $\mathrm{t}, J=7.34 \mathrm{~Hz}), 5.13(1 \mathrm{H}, \mathrm{s}), 3.82,3.44(2 \mathrm{H}, \mathrm{m}), 2.25,2.22(2 \mathrm{H}$, d, $J=16.78 \mathrm{~Hz}), 2.16(3 \mathrm{H}, \mathrm{s}), 2.11,2.07(2 \mathrm{H}, \mathrm{d}, J=16.16 \mathrm{~Hz})$, $0.95(3 \mathrm{H}, \mathrm{s}), 1.00(3 \mathrm{H}, \mathrm{s}), 0.75(3 \mathrm{H}, \mathrm{t}, J=7.08 \mathrm{~Hz}) ;{ }^{13} \mathrm{C} \mathrm{NMR}$ (125 MHz, $\mathrm{CDCl}_{3} / \mathrm{DMSO}_{6}$ ) $\delta 196.0$ (C=O, ketone), 167.8 (C=O, ester), 150.3, 149.8, 143.9, 140.3, 133.8, 133.4, 130.8, 129.7, 129.6, 128.4, 127.7, 126.4, 119.1, 111.8, 106.6, 59.7, 51.1, 40.9, 33.0, 29.5, 27.9, 27.2, 19.1, 14.2. Anal. Calcd for $\mathrm{C}_{30} \mathrm{H}_{30} \mathrm{ClN}_{3} \mathrm{O}_{3}$ : C, 69.82; H, 5.86; N, 8.14. Found: C, 69.67; H, 5.75; N, 8.05. 
2.3.4. Ethyl 4-(1,3-Diphenyl-1H-pyrazol-4-yl)-2-methyl-5-oxo1,4,5,6,7,8-hexahydroquinoline-3-carboxylate (5d). Yellow powder, $\mathrm{mp}=101-103^{\circ} \mathrm{C}$; FT-IR (neat) $\left(v_{\max } / \mathrm{cm}^{-1}\right) 3300$, $3200(\mathrm{NH}), 1690(\mathrm{C}=\mathrm{O}) ;{ }^{1} \mathrm{H}$ NMR $\left(500 \mathrm{MHz}, \mathrm{CDCl}_{3}\right) \delta 7.84$ $(1 \mathrm{H}, \mathrm{s}), 7.82(2 \mathrm{H}, \mathrm{d}, J=7.66 \mathrm{~Hz}), 7.69(2 \mathrm{H}, \mathrm{d}, J=8.33 \mathrm{~Hz})$, $7.42(1 \mathrm{H}, \mathrm{t}, J=7.47 \mathrm{~Hz}), 7.35(1 \mathrm{H}, \mathrm{t}, J=7.32 \mathrm{~Hz}), 7.25(1 \mathrm{H}, \mathrm{t}$, $J=7.38 \mathrm{~Hz}), 6.32(1 \mathrm{H}, \mathrm{s}), 5.30(1 \mathrm{H}, \mathrm{s}), 3.74,4.00(2 \mathrm{H}, \mathrm{m})$, 2.30-2.35 (2H, m), $2.16(3 \mathrm{H}, \mathrm{s}), 1.93,1.84(2 \mathrm{H}, \mathrm{m}), 1.84$, $1.92(2 \mathrm{H}, \mathrm{m}), 1.01(3 \mathrm{H}, \mathrm{t}, J=7.09 \mathrm{~Hz}) ;{ }^{13} \mathrm{C}$ NMR $(125 \mathrm{MHz}$, $\mathrm{CDCl}_{3} / \mathrm{DMSO}_{\mathrm{d}}$ ) $\delta 196.3$ (C=O, ketone), 167.7 (C=O, ester), $152.5,150.6,143.0,140.4,135.3,129.8,129.6,128.4$, 128.2, 127.9, 127.5, 126.4, 119.3, 112.9, 106.6, 60.1, 37.4, 27.6, 27.5, 21.4, 19.4, 14.5. Anal. Calcd for $\mathrm{C}_{28} \mathrm{H}_{27} \mathrm{~N}_{3} \mathrm{O}_{3}:$ C, 74.15; H, 6.00; N, 9.27. Found: C, 74.10; H, 6.26; N, 8.10.

2.3.5. Ethyl 2-Methyl-4-(3-(4-nitrophenyl)-1-phenyl-1H-pyrazol-4-yl)-5-oxo-1,4,5,6,7,8-hexa-hydroquinoline-3-carboxylate (5e). Yellow solid, $\mathrm{mp}=202-203^{\circ} \mathrm{C}$; FT-IR (neat) $\left(v_{\max } / \mathrm{cm}^{-1}\right) 3300,3200(\mathrm{NH}), 1690(\mathrm{C}=\mathrm{O}), 1640(\mathrm{C}=\mathrm{C}$, olefinic), 1510, $1330\left(\mathrm{NO}_{2}\right) ;{ }^{1} \mathrm{H}$ NMR $\left(500 \mathrm{MHz}, \mathrm{CDCl}_{3}\right) \delta 8.31$ $(2 \mathrm{H}, \mathrm{d}, J=8.74 \mathrm{~Hz}), 8.25(2 \mathrm{H}, \mathrm{d}, J=8.74 \mathrm{~Hz}), 8.04(1 \mathrm{H}, \mathrm{s})$, $7.73(1 \mathrm{H}, \mathrm{s}), 7.62(2 \mathrm{H}, \mathrm{d}, J=7.82 \mathrm{~Hz}), 7.37(2 \mathrm{H}, \mathrm{t}, J=7.82 \mathrm{~Hz})$, $7.20(1 \mathrm{H}, \mathrm{t}, J=7.34 \mathrm{~Hz}), 5.22(1 \mathrm{H}, \mathrm{s}), 3.58,3.86(2 \mathrm{H}, \mathrm{m}), 2.37$ $(2 \mathrm{H}, \mathrm{m}), 2.28(2 \mathrm{H}, \mathrm{m}), 2.22(3 \mathrm{H}, \mathrm{s}), 1.84,1.19(2 \mathrm{H}, \mathrm{m}), 0.80$ $(3 \mathrm{H}, \mathrm{t}, J=7.08 \mathrm{~Hz}) ;{ }^{13} \mathrm{C}$ NMR $\left(125 \mathrm{MHz}, \mathrm{CDCl}_{3} / \mathrm{DMSO}-\mathrm{d}_{6}\right)$ $\delta 196.5(\mathrm{C}=\mathrm{O}$, ketone), $167.7(\mathrm{C}=\mathrm{O}$, ester), 151.3, 149.1, 147.3, $144.1,142.2,140.2,130.7,130.0,129.7,128.3,126.8,123.6,119.3$, 113.2, 106.4, 59.9, 37.4, 27.4, 27.3, 21.5, 19.2, 14.4. Anal. Calcd for $\mathrm{C}_{28} \mathrm{H}_{26} \mathrm{~N}_{4} \mathrm{O}_{5}$ : C, 67.46; $\mathrm{H}, 5.26 ; \mathrm{N}, 11.24$. Found: $\mathrm{C}, 67.30 ; \mathrm{H}$, $5.16 ; \mathrm{N}, 11.13$.

2.3.6. Ethyl 4-(3-(4-Chlorophenyl)-1-phenyl-1H-pyrazol-4-yl) -2-methyl-5-oxo-1,4,5,6,7,8-hexa-hydroquinoline-3-carboxylate (5f). White solid, $\mathrm{mp}=257-258^{\circ} \mathrm{C}$; FT-IR (neat) $\left(v_{\max } / \mathrm{cm}^{-1}\right)$ 3300, $3200(\mathrm{NH}), 1690(\mathrm{C}=\mathrm{O}), 1645(\mathrm{C}=\mathrm{C}$, olefinic), $1070(\mathrm{C}-\mathrm{Cl}) ;{ }^{1} \mathrm{H}$ NMR $\left(500 \mathrm{MHz}, \mathrm{CDCl}_{3}\right) \delta 7.92$ $(2 \mathrm{H}, \mathrm{d}, J=8.41 \mathrm{~Hz}), 7.79(1 \mathrm{H}, \mathrm{s}), 7.72(1 \mathrm{H}, \mathrm{s}), 7.61(2 \mathrm{H}, \mathrm{d}, J=$ $7.79 \mathrm{~Hz}), 7.35(2 \mathrm{H}, \mathrm{d}, J=8.41 \mathrm{~Hz}), 7.34(2 \mathrm{H}, \mathrm{t}, J=7.53 \mathrm{~Hz}), 7.18$ $(1 \mathrm{H}, \mathrm{t}, J=7.38 \mathrm{~Hz}), 5.20(1 \mathrm{H}, \mathrm{s}), 3.91,3.59(2 \mathrm{H}, \mathrm{m}), 2.34-2.31$ $(2 \mathrm{H}, \mathrm{m}), 2.29-2.24(2 \mathrm{H}, \mathrm{m}), 2.17(3 \mathrm{H}, \mathrm{s}), 1.91,1.89(2 \mathrm{H}, \mathrm{m})$, $0.87(3 \mathrm{H}, \mathrm{t}, J=7.10 \mathrm{~Hz}) ;{ }^{13} \mathrm{C}$ NMR $\left(125 \mathrm{MHz}, \mathrm{CDCl}_{3} / \mathrm{DMSO}-\right.$ $\left.\mathrm{d}_{6}\right) \delta 196.4(\mathrm{C}=\mathrm{O}$, ketone), $167.9(\mathrm{C}=\mathrm{O}$, ester $), 151.3,150.6$, $143.7,140.4,142.0,133.9,133.6,130.9,129.6,129.5,128.4,127.8$, 126.4, 119.1, 113.0, 106.5, 59.8, 37.5, 27.4, 21.5, 19.1, 14.5. Anal. Calcd for $\mathrm{C}_{28} \mathrm{H}_{26} \mathrm{ClN}_{3} \mathrm{O}_{3}$ : C, 68.92; H, 5.37; N, 8.61. Found: C, 68.74; H, 5.18; N, 8.52.

2.3.7. Ethyl 4-(1,3-Diphenyl-1H-pyrazol-4-yl)-2-methyl-5oxo-4,5-dihydro-1H-indeno[1,2-b]-pyridine-3-carboxylate

(5g). Red solid, $\mathrm{mp}=160-162^{\circ} \mathrm{C}$; FT-IR (neat) $\left(v_{\max } / \mathrm{cm}^{-1}\right)$ 3280, $3180(\mathrm{NH}), 1680,1670(\mathrm{C}=\mathrm{O}), 1635(\mathrm{C}=\mathrm{C}$, olefinic); ${ }^{1} \mathrm{H}$ NMR $\left(500 \mathrm{MHz}, \mathrm{CDCl}_{3}\right) \delta 9.04(1 \mathrm{H}, \mathrm{s}), 7.90(2 \mathrm{H}, \mathrm{d}, J$ $=7.21 \mathrm{~Hz}), 7.76(1 \mathrm{H}, \mathrm{s}), 7.62(2 \mathrm{H}, \mathrm{d}, J=7.92 \mathrm{~Hz}), 7.38-7.27$ $(\mathrm{m}, 7 \mathrm{H}), 7.22-7.14(3 \mathrm{H}, \mathrm{m}), 5.12(1 \mathrm{H}, \mathrm{s}), 3.90,3.67(2 \mathrm{H}, \mathrm{m})$, $2.30(3 \mathrm{H}, \mathrm{s}), 0.79(3 \mathrm{H}, \mathrm{t}, J=7.10 \mathrm{~Hz}) ;{ }^{13} \mathrm{C}$ NMR $(125 \mathrm{MHz}$, $\left.\mathrm{CDCl}_{3} / \mathrm{DMSO}_{-} \mathrm{d}_{6}\right) \delta 192.8(\mathrm{C}=\mathrm{O}$, ketone), $167.8(\mathrm{C}=\mathrm{O}$, ester), $154.2,152.1,143.8,140.4,137.1,134.6,134.5,131.5,130.2,129.6$, $129.5,128.5,128.4,128.0,127.9,126.3,121.1,119.1,118.9,110.1$,
108.7, 60.0, 27.6, 19.3, 14.1. Anal. Calcd for $\mathrm{C}_{31} \mathrm{H}_{25} \mathrm{~N}_{3} \mathrm{O}_{3}$ : C, 76.37; H, 5.17; N, 8.62. Found: C, 76.43; H, 5.10; N, 8.46.

2.3.8. Ethyl 2-Methyl-4-(3-(4-nitrophenyl)-1-phenyl-1H-pyrazol-4-yl)-5-oxo-4,5-dihydro-1H-indeno[1,2-b]pyridine-3-carboxylate $(5 \boldsymbol{h})$. Red solid, $\mathrm{mp}=167-168^{\circ} \mathrm{C}$; FT-IR (neat) $\left(v_{\max } / \mathrm{cm}^{-1}\right) 3300,3180(\mathrm{NH}), 1690,1680(\mathrm{C}=\mathrm{O}), 1640(\mathrm{C}=\mathrm{C}$, olefinic), 1510, $1330\left(\mathrm{NO}_{2}\right) ;{ }^{1} \mathrm{H} \mathrm{NMR}\left(500 \mathrm{MHz}, \mathrm{CDCl}_{3}\right) \delta 9.31$ $(1 \mathrm{H}, \mathrm{s}), 8.21(2 \mathrm{H}, \mathrm{d}, J=8.84 \mathrm{~Hz}), 8.15(2 \mathrm{H}, \mathrm{d}, J=8.84 \mathrm{~Hz})$, $7.77(1 \mathrm{H}, \mathrm{s}), 7.60(2 \mathrm{H}, \mathrm{d}, J=8.58), 7.30-7.37(3 \mathrm{H}, \mathrm{m}), 7.24-7.15$ $(4 \mathrm{H}, \mathrm{m}), 5.08(1 \mathrm{H}, \mathrm{s}), 3.86,3.75(2 \mathrm{H}, \mathrm{m}), 2.33(3 \mathrm{H}, \mathrm{s}), 0.80$ $(3 \mathrm{H}, \mathrm{t}, J=7.10 \mathrm{~Hz}) ;{ }^{13} \mathrm{C}$ NMR $\left(125 \mathrm{MHz}, \mathrm{CDCl}_{3} / \mathrm{DMSO}^{-\mathrm{d}_{6}}\right)$ $\delta 192.7(\mathrm{C}=\mathrm{O}$, ketone), $167.6(\mathrm{C}=\mathrm{O}$, ester $), 154.2,149.5,147.3$, $144.4,141.5,140.1,136.9,134.4,131.6,130.3,130.1,129.7,129.4$, 128.5, 126.9, 123.6, 121.2, 119.2, 119.1, 109.7, 108.2, 60.1, 27.8, 19.4, 14.2. Anal. Calcd for $\mathrm{C}_{31} \mathrm{H}_{24} \mathrm{~N}_{4} \mathrm{O}_{5}$ : C, 69.91; $\mathrm{H}, 4.54 ; \mathrm{N}, 10.52$. Found: $\mathrm{C}, 69.70 ; \mathrm{H}, 4.38 ; \mathrm{N}, 10.34$.

2.3.9. Ethyl 4-(3-(4-Chlorophenyl)-1-phenyl-1H-pyrazol-4-yl)2-methyl-5-oxo-4,5-dihydro-1H-indeno[1,2-b]pyridine-3-carboxylate (5i). Red solid, $\mathrm{mp}=189-191^{\circ} \mathrm{C}$; FT-IR (neat) $\left(v_{\max } / \mathrm{cm}^{-1}\right) 3300,3190(\mathrm{NH}), 1680(\mathrm{C}=\mathrm{O}), 1640(\mathrm{C}=\mathrm{C}), 1090$ $(\mathrm{C}-\mathrm{Cl}) ;{ }^{1} \mathrm{H}$ NMR $\left(500 \mathrm{MHz}, \mathrm{CDCl}_{3}\right) \delta 9.16(1 \mathrm{H}, \mathrm{s}), 7.86(2 \mathrm{H}$, $\mathrm{d}, J=8.86 \mathrm{~Hz}), 7.74(1 \mathrm{H}, \mathrm{s}), 7.59(2 \mathrm{H}, \mathrm{d}, J=7.68 \mathrm{~Hz}), 7.32(2 \mathrm{H}$, d, $J=8.42 \mathrm{~Hz}), 7.30-7.35(3 \mathrm{H}, \mathrm{m}), 7.13-7.26(4 \mathrm{H}, \mathrm{m}), 5.06(1 \mathrm{H}$, s), $3.89,3.73(2 \mathrm{H}, \mathrm{m}), 2.30(3 \mathrm{H}, \mathrm{s}), 0.83(3 \mathrm{H}, \mathrm{t}, J=7.11 \mathrm{~Hz}) ;{ }^{13} \mathrm{C}$ NMR $\left(125 \mathrm{MHz}, \mathrm{CDCl}_{3} / \mathrm{DMSO}_{\mathrm{d}}\right) \delta 192.8(\mathrm{C}=\mathrm{O}$, ketone), 167.7 ( $\mathrm{C}=\mathrm{O}$, ester), 154.2, 150.8, 144.0, 140.2, 137.0, 134.5, 133.7, $133.2,131.6,130.8,130.2,129.6,128.6,128.5,128.1,126.5,121.1$, 119.1, 119.0, 109.9, 108.5, 60.1, 27.7, 19.3, 14.2. Anal. Calcd for $\mathrm{C}_{31} \mathrm{H}_{24} \mathrm{ClN}_{3} \mathrm{O}_{3}: \mathrm{C}, 71.33 ; \mathrm{H}, 4.63 ; \mathrm{N}, 8.05$. Found: C, 71.17; H, $4.45 ; \mathrm{N}, 8.16$.

\section{Results and Discussion}

In continuation of our ongoing interests on the synthesis of 1,4-dihydropyridine derivatives $[29,30]$, we have developed an efficient method for the synthesis of the target products via a one-pot four-component coupling reaction of heteroarylaldehyde (1) (prepared by the reaction of related arylhydrazone and DMF in the presence of 2,4,6-trichloro1,3,5-triazine (TCT)) [28], 1,3-diketone (2), ethylacetoacetate (3), and ammonium acetate in the presence of cellulosesulfuric acid as a biosupported solid acid catalyst (Scheme 1). The reaction gave new derivatives of 1,4-dihydropyridines in lower reaction times (38-45 $\mathrm{min})$ and excellent yields (8595\%) (Table 1).

The reactions (entries $\mathbf{a}, \mathbf{d}$ and $\mathbf{g}$ ) were also carried out in the absence of cellulose-sulfuric acid in the same conditions which furnished the desired products in much longer reaction times (120-125 min) and lower yields (Table 1).

In order to optimize the effect of the amount of catalyst on the efficiency of the reaction, the preparation of $\mathbf{5 a}$ was selected as model reaction under reflux condition. This study gave the optimized amount of the catalyst as $0.05 \mathrm{~g} / \mathrm{mmol}$ of substrate. The effect of different solvents $(\mathrm{EtOH}, \mathrm{MeOH}$, $\mathrm{CH}_{3} \mathrm{CN}, 1,4$-dioxane, and DMF) on the preparation of $\mathbf{5 a}$ showed that EtOH was the solvent of choice. Therefore, 


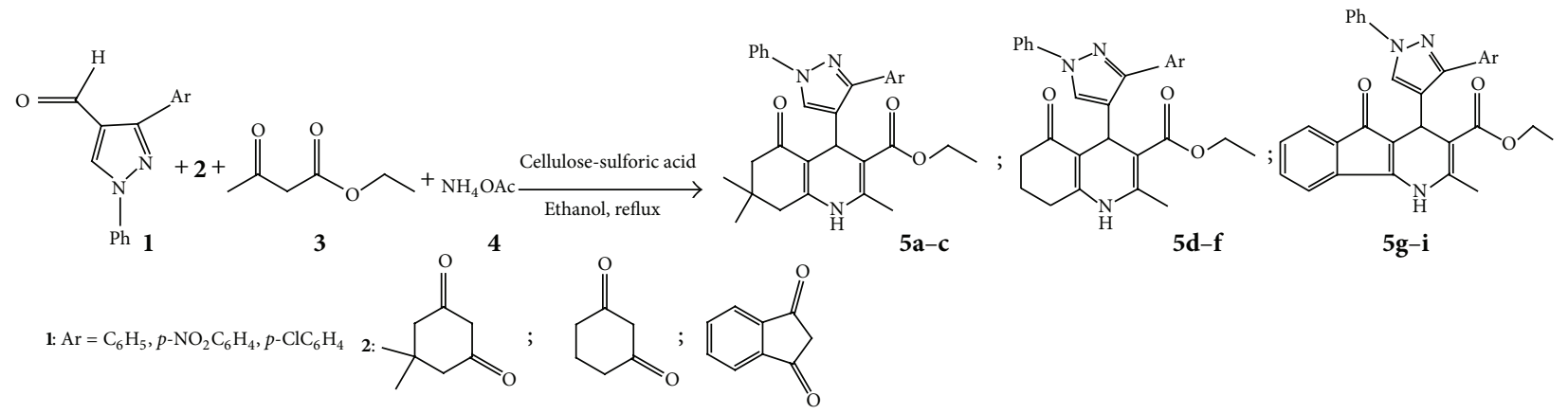

SCHEME 1: Efficient synthesis of fused 1,4-dihydropyridines.

all the reactions described in this report were carried out under optimized conditions (Table 1). The catalyst which was prepared according to the literature reports [31-33] is recoverable and was run for three consecutive cycles, furnishing the product (5a) without loss of catalytic activity. The structures of all products were deduced from ${ }^{1} \mathrm{H}$ NMR, ${ }^{13} \mathrm{C}$ NMR and IR spectral analyses. ${ }^{1} \mathrm{H}$ NMR showed H-4 proton at $5.06-5.33 \mathrm{ppm}$ as singlet which clearly confirmed the formation of 1,4-dihydropyridine moiety.

\section{Conclusions}

In summary, we have developed a convenient and facile protocol for the synthesis of new heteroaryl substituted 1,4dihydropyridines in the presence of cellulose-sulfuric acid as an efficient biosupported solid acid catalyst in lower reaction times (37-45 min) and excellent yields (85-95\%). The simple procedures combined with easy recovery and reuse of the catalyst make this method economic, environmentally benign, and user-friendly process for the synthesis of these biolabile fused 1,4-dihydropyridine derivatives. The method is amenable for iterative combinatorial library generation.

\section{Conflict of Interests}

The authors declare no conflict of interests.

\section{Acknowledgment}

The authors are thankful to the Research Council of University of Guilan for the partial support of this work.

\section{References}

[1] M. Suárez, Y. Verdecia, B. Illescas et al., "Synthesis and study of novel fulleropyrrolidines bearing biologically active 1,4dihydropyridines," Tetrahedron, vol. 59, no. 46, pp. 9179-9186, 2003.

[2] R. Budriesi, A. Bisi, P. Ioan et al., "1,4-Dihydropyridine derivatives as calcium channel modulators: the role of 3-methoxyflavone moiety," Bioorganic and Medicinal Chemistry, vol. 13, no. 10, pp. 3423-3430, 2005.
[3] D. Boschi, G. Caron, S. Visentin et al., "Searching for balanced hybrid NO-donor 1,4-dihydropyridines with basic properties," Pharmaceutical Research, vol. 18, no. 7, pp. 987-991, 2001.

[4] B. K. Singh, M. Mishra, N. Saxena et al., "Synthesis of 2-sulfanyl6-methyl-1,4-dihydropyrimidines as a new class of antifilarial agents," European Journal of Medicinal Chemistry, vol. 43, no. 12, pp. 2717-2723, 2008.

[5] A. Fassihi, Z. Azadpour, N. Delbari et al., "Synthesis and antitubercular activity of novel 4-substituted imidazolyl-2,6dimethyl-N3,N5-bisaryl-1,4-dihydropyridine-3,5-dicarboxamides," European Journal of Medicinal Chemistry, vol. 44, no. 8, pp. 3253-3258, 2009.

[6] S. Gaillard, C. Papamicaël, F. Marsais, G. Dupas, and V. Levacher, "Preparation of 1,4-dihydroquinolines bearing a chiral sulfoxide group: new highly enantioselective recyclable NADH mimics," Synlett, no. 3, pp. 441-444, 2005.

[7] K. Xie, Y.-C. Liu, Y. Cui, J.-G. Wang, Y. Fu, and T. C. W. Mak, "Nmethyl-(R)-3-(tert-butyl)-sulfinyl-1,4-dihydropyridine: a novel NADH model compound," Molecules, vol. 12, no. 3, pp. 415-422, 2007.

[8] J. J. Vanden Eynde and A. Mayence, "Synthesis and aromatization of Hantzsch 1,4-dihydropyridines under microwave irradiation: an overview," Molecules, vol. 8, no. 4, pp. 381-391, 2003.

[9] C. Simon, T. Constantieux, and J. Rodriguez, "Utilisation of 1,3dicarbonyl derivatives in multicomponent reactions," European The Journal of Organic Chemistry, no. 24, pp. 4957-4980, 2004.

[10] B. M. Khadilkar, V. G. Gaikar, and A. A. Chitnavis, "Aqueous hydrotrope solution as a safer medium for microwave enhanced Hantzsch dihydropyridine ester synthesis," Tetrahedron Letters, vol. 36, no. 44, pp. 8083-8086, 1995.

[11] L. Öhberg and J. Westman, "An efficient and fast procedure for the Hantzsch dihydropyridine synthesis under microwave conditions," Synlett, no. 8, pp. 1296-1298, 2001.

[12] A. Agarwal and P. M. S. Chauhan, "Solid supported synthesis of structurally diverse dihydropyrido[2,3-d] pyrimidines using microwave irradiation," Tetrahedron Letters, vol. 46, no. 8, pp. 1345-1348, 2005.

[13] S.-J. Ji, Z.-Q. Jiang, J. Lu, and T.-P. Loh, "Facile ionic liquidspromoted one-pot synthesis of polyhydroquinoline derivatives under solvent free conditions," Synlett, vol. 2004, no. 5, pp. 831$835,2004$.

[14] R. Sridhar and P. T. Perumal, "A new protocol to synthesize 1,4dihydropyridines by using 3,4,5-trifluorobenzeneboronic acid 
as a catalyst in ionic liquid: synthesis of novel 4-(3-carboxyl-1Hpyrazol-4-yl)-1,4-dihydropyridines," Tetrahedron, vol. 61, no. 9, pp. 2465-2470, 2005.

[15] A. P. Phillips, "Hantzsch's pyridine synthesis," The Journal of the American Chemical Society, vol. 71, no. 12, pp. 4003-4007, 1949.

[16] A. G. Anderson Jr. and G. Berkelhammer, "A study of the primary acid reaction on model compounds of reduced diphosphopyridine nucleotide," Journal of the American Chemical Society, vol. 80, no. 4, pp. 992-999, 1958.

[17] H. Singh, D. Dolly, S. Singh Chimni, and S. Kumar, "Acid catalysed enamine induced transformation of 1,3-dimethyl-5formyluracil. A unique annulation reaction with enaminones," Tetrahedron, vol. 51, no. 46, pp. 12775-12780, 1995.

[18] M. F. Gordeev, D. V. Patel, and E. M. Gordon, "Approaches to combinatorial synthesis of heterocycles: a solid-phase synthesis of 1,4-dihydropyridines," The Journal of Organic Chemistry, vol. 61, no. 3, pp. 924-928, 1996.

[19] J. G. Breitenbucher and G. Figliozzi, "Solid-phase synthesis of 4-aryl-1,4-dihydropyridines via the Hantzsch three component condensation," Tetrahedron Letters, vol. 41, no. 22, pp. 4311-4315, 2000.

[20] J.-C. Liang, J.-L. Yeh, C.-S. Wang, S.-F. Liou, C.-H. Tsai, and I.-J. Chen, "The new generation dihydropyridine type calcium blockers, bearing 4-phenyl oxypropanolamine, display $\alpha$ - $/ \beta$-adrenoceptor antagonist and long-acting antihypertensive activities," Bioorganic and Medicinal Chemistry, vol. 10, no. 3, pp. 719-730, 2002.

[21] A. Dondoni, A. Massi, E. Minghini, S. Sabbatini, and V. Bertolasi, "Model studies toward the synthesis of dihydropyrimidinyl and pyridyl $\alpha$-amino acids via three-component Biginelli and Hantzsch cyclocondensations," The Journal of Organic Chemistry, vol. 68, no. 16, pp. 6172-6183, 2003.

[22] A. Dondoni, A. Massi, E. Minghini, and V. Bertolasi, "Multicomponent Hantzsch cyclocondensation as a route to highly functionalized 2- and 4-dihydropyridylalanines, 2- and 4pyridylalanines, and their $\mathrm{N}$-oxides: preparation via a polymerassisted solution-phase approach," Tetrahedron, vol. 60, no. 10, pp. 2311-2326, 2004.

[23] N. Tewari, N. Dwivedi, and R. P. Tripathi, "Tetrabutylammonium hydrogen sulfate catalyzed eco-friendly and efficient synthesis of glycosyl 1,4-dihydropyridines," Tetrahedron Letters, vol. 45, no. 49, pp. 9011-9014, 2004.

[24] J. D. Moseley, "Alternative esters in the synthesis of ZD0947," Tetrahedron Letters, vol. 46, no. 18, pp. 3179-3181, 2005.

[25] G. Sabitha, G. S. K. K. Reddy, C. S. Reddy, and J. S. Yadav, "A novel TMSI-mediated synthesis of Hantzsch 1,4-dihydropyridines at ambient temperature," Tetrahedron Letters, vol. 44, no. 21, pp. 4129-4131, 2003.

[26] L.-M. Wang, J. Sheng, L. Zhang et al., "Facile Yb(OTf) promoted one-pot synthesis of polyhydroquinoline derivatives through Hantzsch reaction," Tetrahedron, vol. 61, no. 6, pp. 1539-1543, 2005.

[27] S. Kikuchi, M. Iwai, H. Murayama, and S.-I. Fukuzawa, "Catalytic synthesis of 1,4-dihydropyridine derivatives using scandium(III) triflate," Tetrahedron Letters, vol. 49, no. 1, pp. 114-116, 2008.

[28] L. De Luca, G. Giacomelli, S. Masala, and A. Porcheddu, "A mild procedure for the preparation of 3-aryl-4-formylpyrazoles," Synlett, no. 13, pp. 2299-2302, 2004.

[29] M. Nikpassand, M. Mamaghani, F. Shirini, and K. Tabatabaeian, "A convenient ultrasound-promoted regioselective synthesis of fused polycyclic 4-aryl-3-methyl-4,7-dihydro-1H-pyrazolo[3,4b]pyridines," Ultrasonics Sonochemistry, vol. 17, no. 2, pp. 301305, 2010.

[30] M. Nikpassand, M. Mamaghani, and K. Tabatabaeian, "An efficient one-pot three-component synthesis of fused 1,4-dihydropyridines using HY-zeolite," Molecules, vol. 14, no. 4, pp. 1468-1474, 2009.

[31] A. Shaabani and A. Maleki, "Cellulose sulfuric acid as a bio-supported and recyclable solid acid catalyst for the onepot three-component synthesis of $\alpha$-amino nitriles," Applied Catalysis A, vol. 331, no. 1, pp. 149-151, 2007.

[32] A. Shaabani, A. Rahmati, and Z. Badri, "Sulfonated cellulose and starch: new biodegradable and renewable solid acid catalysts for efficient synthesis of quinolines," Catalysis Communications, vol. 9, no. 1, pp. 13-16, 2008.

[33] A. Rahmatpour, "Cellulose sulfuric acid as a biodegradable and recoverable solid acid catalyst for one pot synthesis of substituted pyrroles under solvent-free conditions at room temperature," Reactive and Functional Polymers, vol. 71, no. 1, pp. 80-83, 2011. 

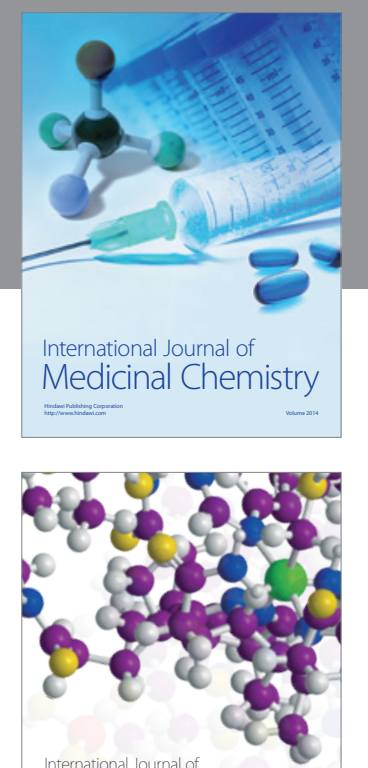

\section{Carbohydrate} Chemistry

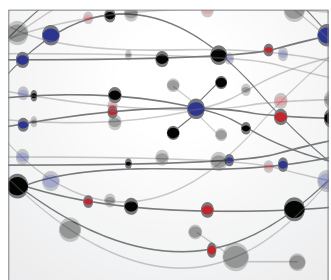

The Scientific World Journal
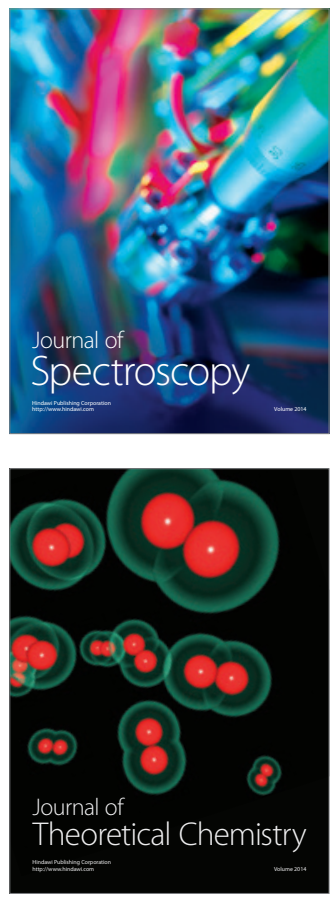
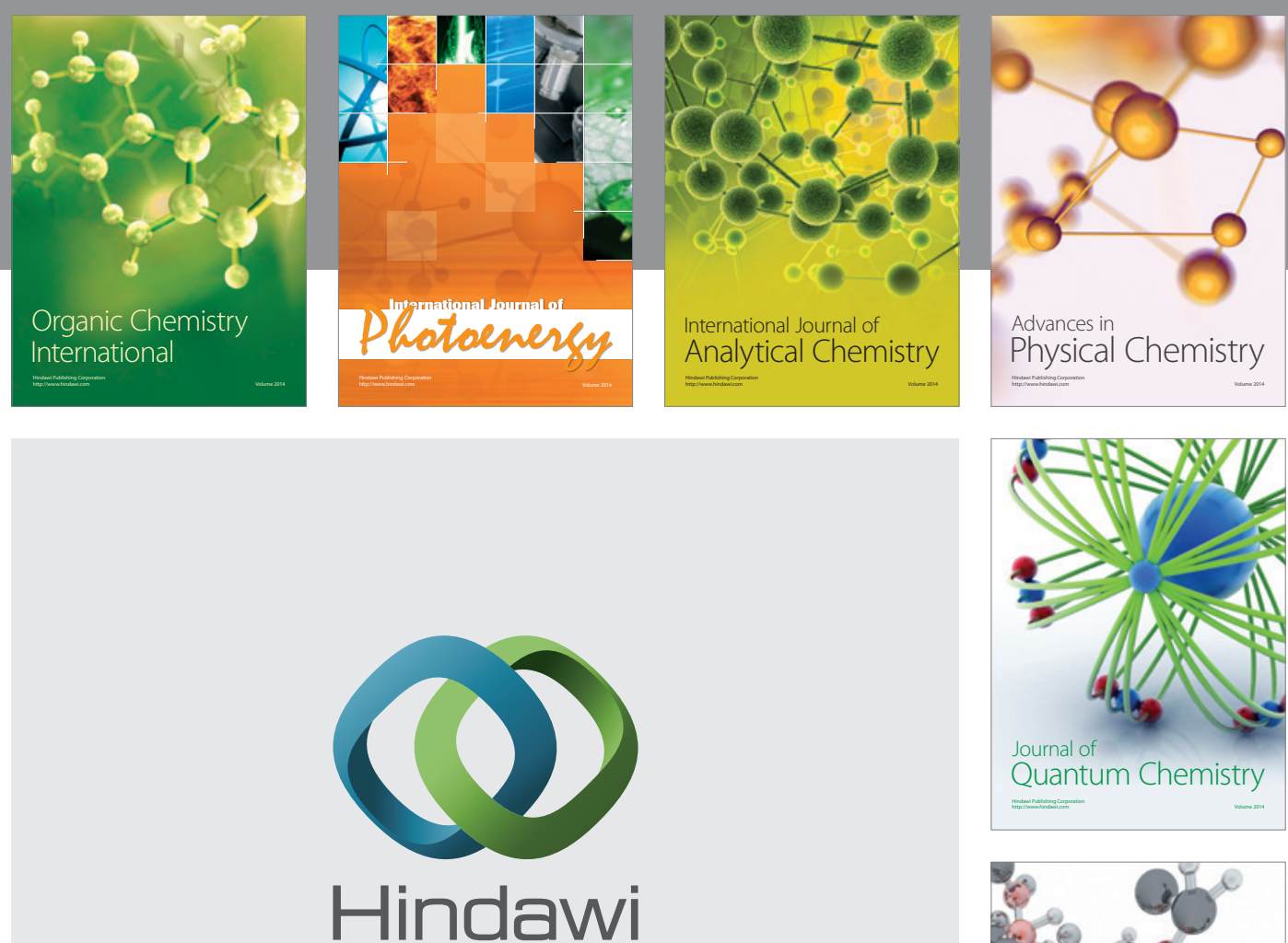

Submit your manuscripts at

http://www.hindawi.com

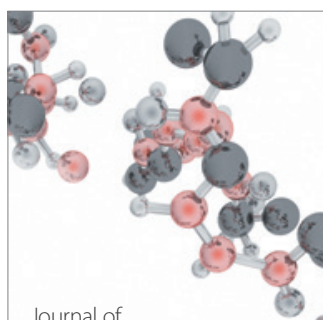

Analytical Methods

in Chemistry

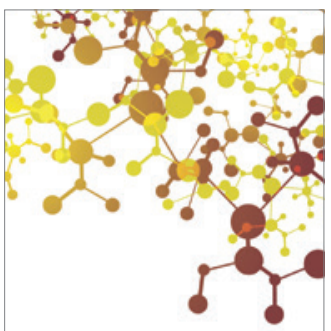

Journal of

Applied Chemistry

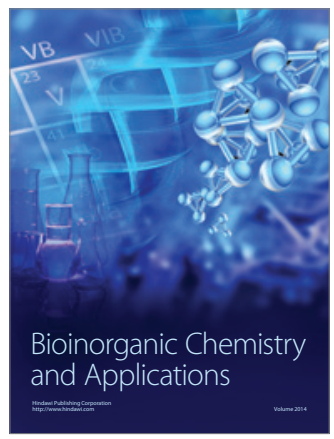

Inorganic Chemistry
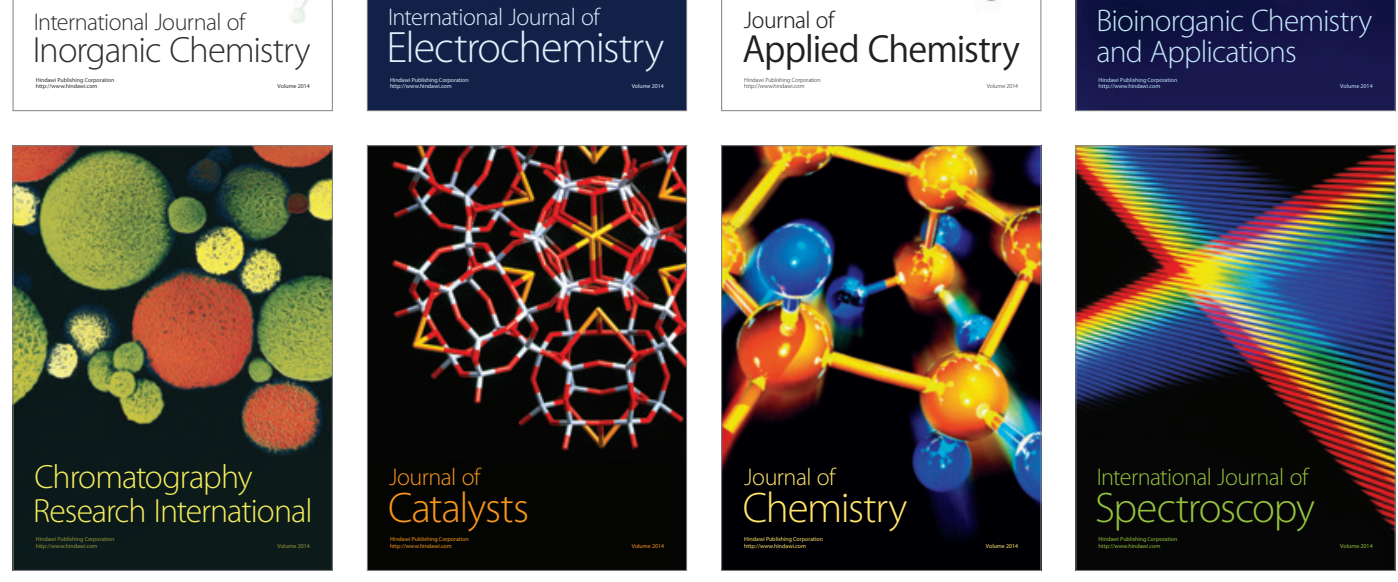